\title{
The Effect of Cardiovascular Responses after Aerobic Exercise in Menstrual Cycle
}

\author{
Joonsung Park, Mi-Song Kim, Hosung Nho, Kyung-Ae Kim, Jong-Kyung Kim, Hyun-Min Choi* \\ Graduate School of Physical Education, Kyung Hee University, Yongin, South Korea \\ Email: ^hmchoi92@khu.ac.kr
}

How to cite this paper: Park, J., Kim, M.-S., Nho, H., Kim, K.-A., Kim, J.-K. and Choi, H.-M. (2017) The Effect of Cardiovascular Responses after Aerobic Exercise in Menstrual Cycle. Health, 9, 425-434. https://doi.org/10.4236/health.2017.93030

Received: January 8, 2017

Accepted: March 7, 2017

Published: March 10, 2017

Copyright $\odot 2017$ by authors and Scientific Research Publishing Inc. This work is licensed under the Creative Commons Attribution International License (CC BY 4.0).

http://creativecommons.org/licenses/by/4.0/

\begin{abstract}
Purpose: We investigated the effect of menstrual cycle on the cardiovascular responses during recovery period from exercise. 10 healthy women were participated in the early follicular phase (EP: low estrogen and low progesterone: 1 - 4 days) and the late follicular phase (LP: high estrogen and low progesterone: 10 - 13 days) during menstrual cycle. Methods: All subjects completed a graded cycling exercise testing to determine the relative exercise intensity. All subjects were assessed systolic blood pressure (SBP), diastolic blood pressure (DBP), mean arterial pressure (MAP), heart rate (HR), stroke volume (SV), cardiac output (CO), and total vascular conductance (TVC) at resting and during recovery period from moderated cycling exercise during EP and LP. Results: During EP, compared with LP, resting SBP and MAP were higher and TVC was lower $(p<0.05)$. During recovery from exercise, SBP and MAP were higher and TVC lower during EP compared with LP $(p<0.05)$. There were no differences in HR, SV and CO in both phases. Conclusions: This study could be increased the peripheral vasoconstriction in low estrogen levels. And during early follicular phase with low estrogen level, it may maintain an increased blood pressure during recovery period following cycling. We suggest that the timing of the menstrual cycle should be considered when measuring blood pressure during clinical exercise testing.
\end{abstract}

\section{Keywords}

Early Follicular Phase, Late Follicular Phase, Estrogen,

Cardiovascular Response, Healthy Women

\section{Introduction}

Menopause intensifies the risk of cardiovascular disease drastically in women compared with men of the same age by increasing low-density lipoprotein cholesterol (LDLC) levels and blood pressure [1]. For this reason, measures have been devised 
in attempts to improve and prevent cardiovascular disease in women, and of these, participation in physical activity is the most effective and recommended option [2]. Both estrogen and progesterone levels are at their lowest during menstruation, while the level of estrogen secretion increases in the ovulatory phase and estrogen levels drop as progesterone levels rise after ovulation [3].

Changes in women's hormone levels during exercise induce differences in physiological responses [1]. Increasing blood pressure is a cardiovascular response to exercise as workout intensity increases, followed by rises in heart rate (HR) and stroke volume (SV), cardiac output (CO) and total peripheral resistance (TPR) [4]. Changes in cardiovascular responses during exercise according to estrogen level changes are commonly observed in postmenopausal women. Estrogen production is reduced after menopause, and postmenopausal women are characterized by excess cardiovascular responses when doing aerobic exercise and excessive elevation of blood pressure, in particular [5]. Moreover, a difference in response to exercise can be also induced during the menstrual cycle with estrogen level changes in women before menopause [6].

Reduction in estrogen secretion during menstruation activates the sympathetic nervous system and this can lead to vascular contraction by increasing sensitivity to norepinephrine and $\alpha$-adrenergic receptors and excessive blood pressure response during exercise by increasing TPR [1] [7]. On the contrary, raised estrogen levels in the ovulatory phase lower blood pressure during exercise by inducing vascular relaxation through activation of nitric oxide which is a vascular relaxant factor produced by vascular endothelial cells [7]. Likewise, the difference in estrogen levels between menstruation and ovulation generates a difference in cardiovascular responses during exercise [6]. Since excessive elevation of blood pressure may incur heart attack, caution is warranted in hypertensive and prehypertensive adults undergoing exercise [8]. However, no studies have yet been undertaken to investigate cardiovascular responses in the recovery period after exercise according to the menstrual cycle.

Cardiovascular responses in the recovery period after exercise are used in predicting cardiovascular disease such as coronary artery disease. In particular, recovery responses of HR and blood pressure are identified as important predictors, and the longer the recovery time after exercise, the higher the cardiovascular disease risk [9]. Post-exercise cardiovascular responses in recovery are a decrease in $\mathrm{CO}$ and $\mathrm{BP}$ following a reduction in $\mathrm{HR}$ and $\mathrm{SV}$ to restore our body to the pre-exercise resting level [10]. This is resulted from reduction in venous return as the termination of muscle contraction is followed by no action of muscle pump after completing exercise [11]. Furthermore, diminished activation of the sympathetic nervous system upon completion of exercise not only has an impact on the reduction of blood pressure by lowering the operating point of baroreceptor, but also leads to decreased blood pressure as reactivation of the vagus nerves is promoted and HR reduction response is lowered drastically [12] [13]. A more rapid blood pressure reduction during recovery after exercise in women than in men is caused by a drastic reduction in $\mathrm{CO}$ following post-exercise reduction 
in SV and diminished increase in TPR [11]. For these reasons, the risk of orthostatic hypotension, syncope and other conditions immediately following exercise is higher in women than in men [14].

Until now, previous studies have focused on cardiovascular responses during exercise and comparative studies on post-exercise recovery between men and women have been conducted only regardless of the menstrual cycle. Thus, there is a need of review on cardiovascular responses in the recovery phase immediately following aerobic exercise according to estrogen levels in premenopausal women. This study investigated cardiovascular responses in recovery after aerobic exercise between the menstruation and ovulation phases in women between 20 s and 30 s having a regular menstrual cycle.

\section{Methods}

Female students between 20s and 30s attending Kyung Hee University were included as the study subjects who had normal periods for more than six months without a history of use of oral contraceptives and hormones, smoking, gravidity and parity, hypertension, cardiovascular disease, exercise habits, and irregular menstrual cycle. For this study, we were recruited 10 healthy women with regular menstrual cycle from students on university campus. The subjects were instructed to refrain from consuming any alcohol or caffeine-containing drinks for 24 hours prior to the study, and were also asked to avoid strenuous physical activity or intense exercise for 24 hours before participating in the study. On the day of the survey, all participants were fully informed about the content and procedures of the research and consented to the participation. They were asked to complete the health history questionnaire (HHQ), and were fully informed about the items, steps and cautions of the survey. The physical characteristics of the subjects are shown in Table 1.

The menstrual cycle was examined in each participant one month prior to the

Table 1. Physical characteristics of subjects.

\begin{tabular}{cccc}
\hline Variables & \multicolumn{3}{c}{ Participants $(\mathrm{n}=10)$} \\
Age $(\mathrm{yrs})$ & 25.0 & \pm & 2.0 \\
Height $(\mathrm{cm})$ & 164.5 & \pm & 1.5 \\
Body weight $(\mathrm{kg})$ & 54.5 & \pm & 0.9 \\
BMI $\left(\mathrm{kg} / \mathrm{m}^{2}\right)$ & 20.2 & \pm & 0.5 \\
Body fat $(\%)$ & 24.2 & \pm & 1.0 \\
SBP $(\mathrm{mmHg})$ & 109.0 & \pm & 1.0 \\
DBP $(\mathrm{mmHg})$ & 71.0 & \pm & 1.0 \\
MAP $(\mathrm{mmHg})$ & 101.0 & \pm & 0.4 \\
VO & 33.1 & \pm & 2.3 \\
Menstrual $(\mathrm{ml} / \mathrm{kg} / \mathrm{min})$ & 30.0 & \pm & 0.5 \\
Menstrual period (day) & 6.0 & \pm & 0.5
\end{tabular}

Values are expressed as the mean \pm standard error; BMI: body mass index, SBP: systolic blood pressure, DBP: diastolic blood pressure, MAP: mean arterial pressure, HR: heart rate, $\mathrm{VO}_{2 \text { peak: }}$ : peak oxygen uptake. 
study by identifying cycle length and duration of menstruation, and women previously having periods on a regular basis (with an average menstrual cycle of 28 35 days) over six months were included. Measurement was done between the second and fourth days of menstruation by counting the first day of one period as day 1 , and between cycle days 10 - 13 of ovulation after the first day of menstruation [15] [16]. Bioimpedance method (Inbody 3.0) was used to determine body composition by measuring fat mass, lean body mass, percent body fat and body mass index.

\subsection{Body Composition and Resting Blood Pressure Measurement}

Resting blood pressure was measured using sphygmomanometer (Japan) by applying the cuff after given a rest period of 10 minutes in a sitting position. Systolic blood pressure (SBP) and diastolic blood pressure (DBP) were measured twice at a 5-minute interval by placing the stethoscope over the brachial artery, and the minimum values were used.

\subsection{Graded Exercise Loading Test}

Relative exercise intensities were estimated using a gas analyzer (Ultima CPX, USA) and a cycle ergometer (Monark 818, Sweden) The Bruce ramp protocol [17] was used for incremental exercise test at a rate of $60 \mathrm{rpm}$ starting at 0 watt for an initial two minutes as warm-up and by increasing 15 watts every minute. No further increase of $\mathrm{VO}_{2}\left(\mathrm{VO}_{2}<150 \mathrm{ml} / \mathrm{min}\right)$ despite an increase in exercise intensity during the test, $90 \%$ age-predicted maximal $\mathrm{HR}$, an respiratory exchange ratio greater than 1.15 , and a rating of perceived exertion (RPE) equal to or greater than 17 recorded every minute were used as criteria. $\mathrm{VO}_{2 \text { peak }}$ was considered to have occurred when two or more of the above criteria were met [18].

\subsection{Cardiovascular Responses Measure}

Cardiovascular responses were examined using thoracic impedance cardiography (physio flow PF-05, Manatec biomedical, France). HR, SV and CO at rest, during exercise and in recovery were measured in the menstruation and ovulation phases. The subjects were given a 5-minute sitting rest period on the cycle by placing six $30 \mathrm{~mm}$ monitoring electrode patches on the chest. The warm-up was conducted at 0 watt for 2 minutes at $60 \mathrm{rpm}$, and then the subjects performed a 30minute cycling bout at $60 \% \mathrm{VO}_{2 \text { peak }}$ on exercise stress test. After completing cycling exercise, cardiovascular responses were measured in the recovery period. Blood pressure responses were measured every 5 minutes in the recovery period. Mean blood pressure $[\mathrm{MAP}=1 / 3 \times(\mathrm{SBP}-\mathrm{DBP})+\mathrm{DBP}]$ was estimated using measured SBP and DBP, and TVC $[$ TVC $=(\mathrm{CO} \times 1000) / \mathrm{MAP}]$ was calculated using MAP and CO.

\subsection{Data Processing}

All statistical analyses were performed using SPSS Statistics version 21.0. Mean \pm standard error was obtained in each item and the paired t-test was conducted to 
compared the difference in variables between the EP and LP. Comparative analysis was conducted with significance level at 0.05 .

\section{Results}

Figure 1 shows SBP, DBP and MAP during recovery after aerobic exercise in the the EP and LP. SBP and MAP at rest and during exercise were higher during the LP compared to the EP $(p<0.05)$. These values had a significant difference at 5 , 10 and 15 minutes of rest after exercise between the two phases, and increased during the $\mathrm{EP}(p<0.05)$. On the other hand, no difference was found at 20,25 and 30 minutes of rest after exercise between the two phases. DBP had no difference at rest, during exercise and in recovery between the two phases.

Figure 2 shows HR, SV, CO and TVC during recovery after aerobic exercise in the EP and LP. No differences were found in HR, SV and CO either at rest and during exercise or during post-exercise recovery between the two phases. TVC at rest and during exercise had a significant difference between the two phases,
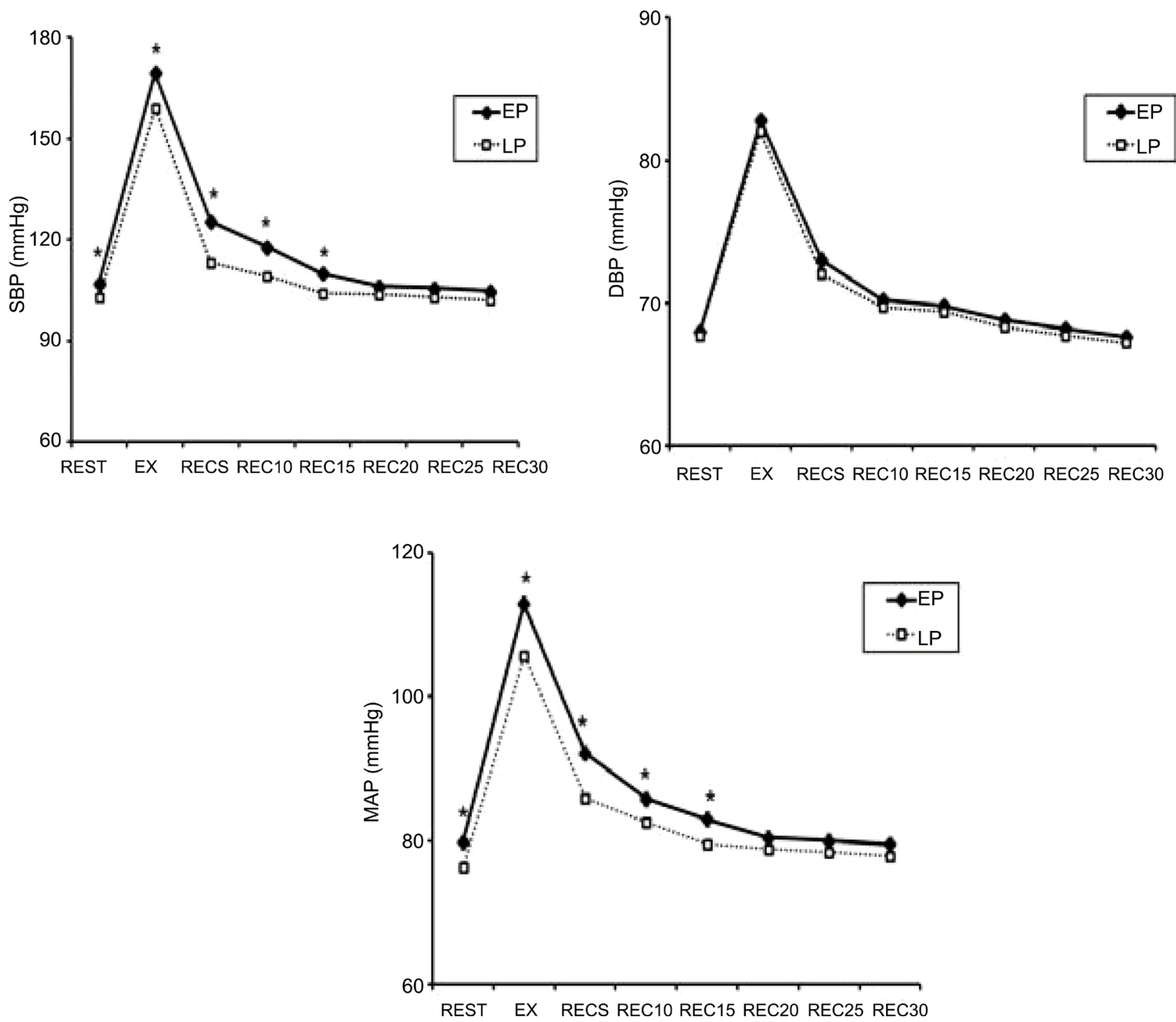

Figure 1. SBP, DBP, MAP responses between EP and LP after aerobic exercise recovery. 

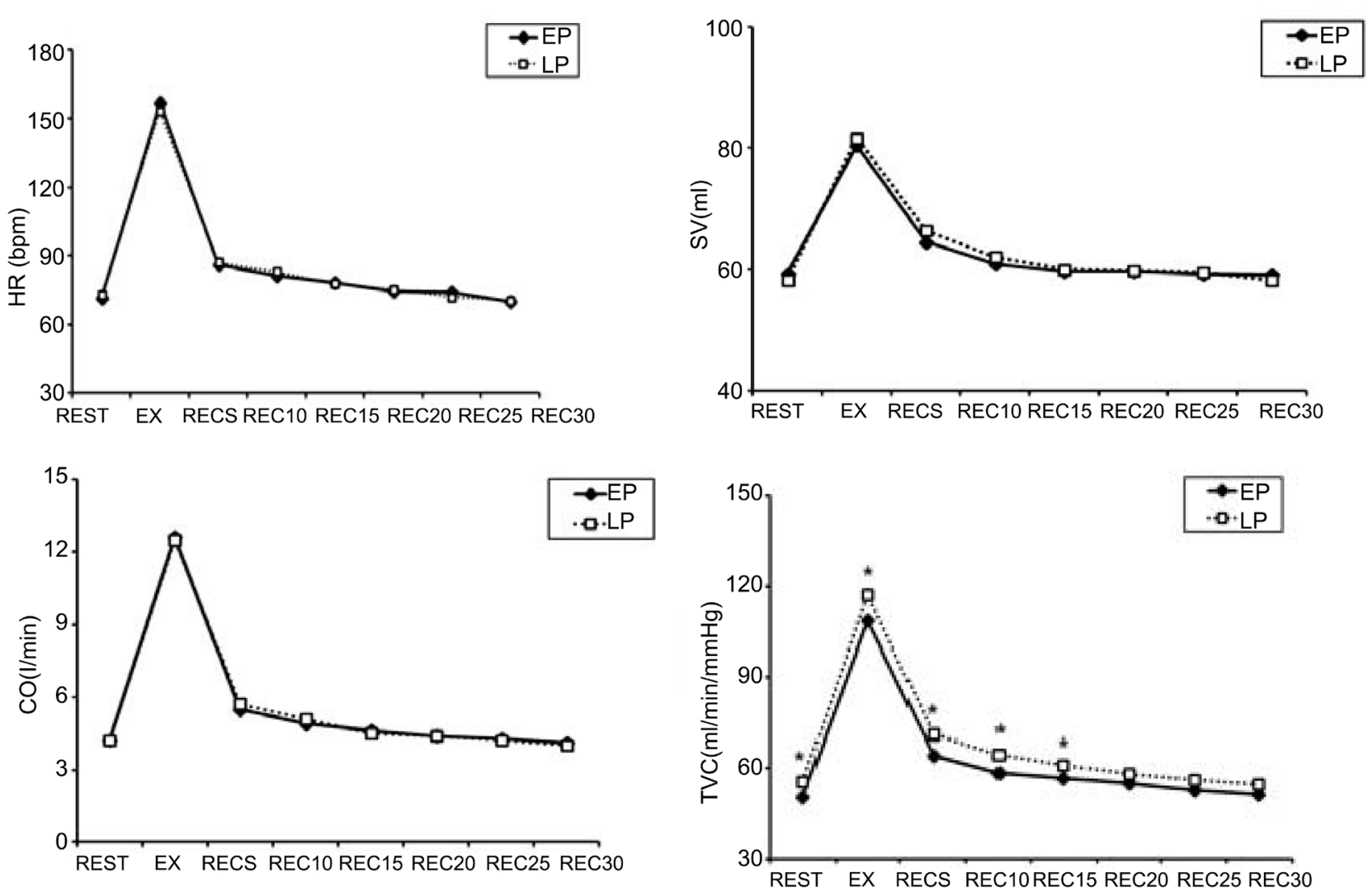

Figure 2. HR, SV, CO, TVC responses during EP and LP after aerobic exercise recovery.

and was elevated in the LP compared to the EP $(p<0.05)$. Moreover, TVC during post-exercise recovery showed an increase in the LP at 5, 10 and 15 minutes of rest. On the contrary, there was no difference at 20, 25 and 30 minutes of rest after exercise.

\section{Discussion}

In the menstrual cycle, estrogen levels are the lowest during menstruation and, during this period, blood pressure responses are relatively greater than during ovulation at rest, a mild-intensity of $40 \% \mathrm{VO}_{2 \text { peak }}$ and a moderate-intensity of $60 \% \mathrm{VO}_{\text {2peak }}[8]$. Decreased estrogen levels during menstruation influence an increase of peripheral resistance as the sympathetic nervous system is more activated and peripheral blood vessels are more contracted [1]. At ovulation, increased estrogen levels not only affect vascular relaxation by triggering the activation of nitric oxide, the vascular relaxant factor, but also are attributable to a lower blood pressure during exercise than at menstruation by having a direct relaxant effect on the smooth muscles [19]. Until now, multiple studies have demonstrated cardiovascular responses during exercise according to the menstrual cycle. However, only a few studies have explored cardiovascular responses in the recovery period after exercise. Therefore, this study aimed to examine cardiovascular responses during post-exercise recovery according to menstrual cycle phase.

Blood pressure is affected by $\mathrm{CO}=\mathrm{HR} \times \mathrm{SV}$ and TPR. In the present study, 
SBP and MAP at rest were significantly higher in the menstruation phase than in the ovulation phase. $\mathrm{CO}$ at rest showed no significant difference between the two phases, but TVC was significantly higher in the ovulation phase. A higher blood pressure at menstruation is thought to be attributable to an increase in TPR. This study was able to verify the fact that estrogen plays an important role in increasing blood pressure by affecting vascular contraction. In this investigation, all subjects underwent aerobic exercise for 30 minutes at an intensity of $60 \%$ $\mathrm{VO}_{2 \text { peak. }}$. As results, SBP, MAP and TVC had a significant difference, while CO demonstrated no change. This outcome is consistent with results from previous studies on cardiovascular responses after mild- and moderate-intensity aerobic exercise according to the menstrual cycle by [6] and others. Furthermore, this study reconfirmed the results of previous studies by [8] who found that $\mathrm{CO}$ increased linearly with increasing exercise intensity and had no difference between the two phases.

In the present study, elevated blood pressure responses during exercise in the menstruation phase compared to the ovulation phase are assumed to be influenced by TPR, equally at rest. Low estrogen levels during menstruation typically result in contraction of peripheral blood vessels by activating the sympathetic nervous system [1]. In addition, norepinephrine levels, an indicator of sympathetic activity, are higher in the menstruation phase than in the ovulation phase [20]. Whereas, the increased levels of estrogen in the ovulation phase induce vascular relaxation by directly stimulating vascular endothelial cells and also decrease BP during exercise through relaxation of peripheral blood vessels by promoting activation of nitric oxide which is a vascular relaxant factor [8] [19]. Thus, an increase of peripheral resistance following decreased estrogen production seems to be one of the causes for a more excessive increase of blood pressure at rest and during exercise in the menstruation phase than in the ovulation phase.

Cardiovascular responses return to resting levels immediately after exercise [21]. Within 5 minutes after stopping exercise, the rapid reduction in $\mathrm{CO}$ is seen as HR and SV decrease [10] [13]. Moreover, cardiovascular responses return to resting levels as vascular resistance is reduced [22]. In the present study, a decrease in all variables was observed after completing exercise, and SBP and MAP, in particular, showed a significant difference at 5, 10 and 15 minutes after stopping exercise between the menstruation and ovulation phases. However, despite a higher tendency in the menstruation phase, no significant difference was found at 20, 25 and 30 minutes of rest after exercise between the two phases.

Although the most dramatic decrease in HR, SV and CO was observed within 5 minutes after completing exercise, there was no significant difference between the two phases in this study. The results aligned with the findings of previous studies on HR, SV and CO in menstrual cycle phases by [23]. Cardiac output increase at start of exercise and sudden reduction after stopping exercise is attributable to a decrease in SV and HR. The autonomic nervous system regulates HR. Upon completion of exercise, HR returns to the resting level with decreased 
sympathetic function and increased parasympathetic stimulation [24]. Because of estrogen production, HR is relatively lower in women than in men, and it is lowest during the ovulation phase as estrogen affects the parasympathetic nervous system [25].

After exercise, SV is reduced as suspended muscle contraction which helps to pump blood decreases venous return [22]. Even though SV varies according to gender and physical strength [26], it is not affected by the menstrual cycle [23]. Although there is no difference in $\mathrm{CO}$ according to menstrual cycle phase, previous studies have reported a higher risk of dizziness or orthostatic hypotension immediately after stopping exercise during the ovulation phase because of a relatively lower blood pressure during ovulation with an effect of peripheral resistance $(22,23)$. TVC showed a significant difference at 5, 10 and 15 minutes after exercise between the two phases, but there was no difference at 20,25 and 30 minutes after exercise.

Vascular conductance is greater during ovulation than during menstruation [14]. Post-exercise TPR is higher during menstruation than during ovulation [23]. In the present study, the time period of statistically significant difference in TVC between the two phases aligned with the time periods of statistically significant difference in SBP and MAP. During post-exercise recovery of blood pressure to the normal level, a more significant reduction was observed in the ovulation phase with an influence of TVC. This finding is anticipated to be attributable to a greater decrease of TPR during ovulation. This investigation found out that the reduction of estrogen levels elevates blood pressure both during exercise and throughout the recovery period by affecting the peripheral blood vessels.

\section{Conclusion}

This study examined cardiovascular responses in the recovery period after aerobic exercise according to menstrual cycle phases with different estrogen levels among women in 20s and 30s. As results, blood pressure responses increased by TPR were found in the recovery period after aerobic exercise during menstruation with decreased estrogen levels compared with that during ovulation.

\section{References}

[1] Knowlton, A.A. and Lee, A.R. (2012) Estrogen and the Cardiovascular System. Pharmacology and Therapeutics, 135, 54-70. https://doi.org/10.1016/j.pharmthera.2012.03.007

[2] Shortreed, S.M., Peeters, A. and Forbes, A.B. (2013) Estimating the Effect of LongTerm Physical Activity on Cardiovascular Disease and Mortality: Evidence from the Framingham Heart Study. Heart, 99, 649-654. https://doi.org/10.1136/heartjnl-2012-303461

[3] Xanne, A.K. and Janse, D.J. (2003) Effects of the Menstrual Cycle on Exercise Performance. Sports Medicine, 33, 883-851.

[4] Power, S.K. and Howley, E.T. (2001) Exercise Physiology: Theory and Application to Fitness and Performance. 6th Edition, Life Science Publishing Co., Seoul.

[5] Harvey, P.J., Morris, B.L., Kubo, T., Picton, P.E., Su, W.S., Notarius, C.F., et al. 
(2005) Hemodynamic After-Effects of Acute Dynamic Exercise in Sedentary Normotensive Postmenopausal Women. Journal of Hypertension, 23, 285-292. https://doi.org/10.1097/00004872-200502000-00010

[6] Hartwich, D., Aldred, S. and Fisher, J.P. (2013) Influence of Menstrual Cycle Phase on Muscle Metaboreflex Control of Cardiac Baroreflex Sensitivity, Heart Rate and Blood Pressure in Humans. Experimental Physiology, 98, 220-232. https://doi.org/10.1113/expphysiol.2012.066498

[7] Adkisson, E.J., Casey, D.P., Beck, D.T., Gurovich, A.N., Martin, J.S. and Braith, R.W. (2010) Central, Peripheral and Resistance Arterial Reactivity: Fluctuates During the Phases of the Menstrual Cycle. Experimental Biology and Medicine, 235, 111-118. https://doi.org/10.1258/ebm.2009.009186

[8] Choi, H.M., Charles, L.S., Nho, H., Kim, M.S., Chang, M.J. and Kim, J.K. (2013) Effects of Ovarian Cycle on Hemodynamic Responses During Dynamic Exercise in Sedentary Women. Korean Journal of Physiology and Pharmacology, 17, 499-503. https://doi.org/10.4196/kjpp.2013.17.6.499

[9] Laukkanen, J.A., Sudhir, K., Ritta, S., Timo, A.L., Rainer, R. and Jukka, T.S. (2004) Systolic Blood Pressure During Recovery from Exercise and the Risk of Acute Myocardial Infarction in Middle-Aged Men. Hypertension, 44, 820-825. https://doi.org/10.1161/01.HYP.0000148460.95060.f2

[10] Carter III, R., Watenpaugh, D.E., Wasmund, W.L., Wasmund, S.L. and Smith, M.L. (1999) Muscle Pump and Central Command During Recovery from Exercise in $\mathrm{Hu}$ mans. Journal of Applied Physiology, 87, 1463-1469.

[11] Carter, R., Watenpaugh, D.E. and Smith, M.L. (2001) Gender Differences in Cardiovascular Regulation during Recovery From Exercise. Journal of Applied Physiology, 91, 1902-1907.

[12] Kulics, J.M., Collins, H.L. and DiCarlo, S.E. (1999) Postexercise Hypotension Is Mediated by Reductions in Sympathetic Nerve Activity. American Journal of Physiology, 276, H27-H32.

[13] Halliwill, J.R. (2001) Mechanisms and Clinical Implications of Post-Exercise Hypotension in Humans. Exercise and Sport Sciences Reviews, 2, 65-70. https://doi.org/10.1097/00003677-200104000-00005

[14] Lynn, B.M., McCord, J.L. and Halliwill, J.R. (2007) Effects of the Menstrual Cycle and Sex on Postexercise Hemodynamics. American Journal of Physiology-Regulatory, Integrative and Comparative Physiology, 292, R1260-R1270.

[15] Ettinger, S.M., Silber, D.H., Gray, K.S., Smith, M.B., Yang, Q.X. and Kunselman, A.R. (1998) Effects of the Ovarian Cycle on Sympathetic Neural Outflow During Static Exercise. Journal of Applied Physiology, 85, 2075-2081.

[16] Boron, W.F. and Boulpaep, E.L. (2003) Medical Physiology: A Cellular and Molecular Approach. Saunders, Philadelphia.

[17] Nho, H., Tanaka, K., Kim, H.S., Watanabe, Y. and Hiyama, T. (1998) Exercise Training in Female Patients with a Family History of Hypertension. European Journal of Applied Physiology, 78, 1-6. https://doi.org/10.1007/s004210050379

[18] American College of Sports Medicine (2009) ACSM's Guidelines for Exercise Testing and Prescription. 8th Edition, Williams \& Wilkins, Seattle.

[19] Jean, F.A., Coralie, F., Audrey, B.G., Julie, F., Henrik, L., Francoise, L., et al. (2010) Estrogen Receptors and Endothelium. Arteriosclerosis Thrombosis and Vascular Biology, 30, 1506-1512. https://doi.org/10.1161/ATVBAHA.109.191221

[20] De Cree, C. (2011) Cathecholamine Responses to Acute and Chronic Exericse According to Mestrural Status. Medicine and Science in Sports and Exercise, 43, 1132. 
https://doi.org/10.1249/MSS.0b013e318214f936

[21] Antonio, C., Valentina, O., Franco, M., Filippo, T. and Alberto, C. (2003) Hemodynamics during Active and Passive Recovery from a Single Bout of Supramaximal Exercise. European Journal of Applied Physiology, 89, 209-216. https://doi.org/10.1007/s00421-003-0796-4

[22] Carter, J.R., Fu, Q., Minson, C.T. and Joyner, M.J. (2013) Ovarian Cycle and Sympathoexcitation in Premenopausal Women. Hypertension, 61, 395-399. https://doi.org/10.1161/HYPERTENSIONAHA.112.202598

[23] Esformes, J.I., Norman, F., Sigley, J. and Birch, K.M. (2006) The Influence of Menstrual Cycle Phase upon Postexercise Hypotension. Medicine and Science in Sports and Exercise, 38, 484-491. https://doi.org/10.1249/01.mss.0000193559.98095.ea

[24] Paolo, T., Federico, S. and Giosue, G. (2001) Post-Exercise Recovery of Autonomic Cardiovascular Control: A Study by Spectrum and Cross-Spectrum Analysis in $\mathrm{Hu}-$ mans. European Journal of Applied Physiology, 84, 187-194. https://doi.org/10.1007/s004210170003

[25] Makoto, T., Masayoshi, S., Shino, U. and Toshiaki, N. (2003) Influence of Menstrual Cycle on Baroreflex Control of Heart Rate: Comparison with Male Volunteers. American Journal of Physiology-Regulatory, Integrative and Comparative Physiology, 258, R1019-R1097.

[26] Senitk, A.N., Charkoudian, N. and Halliwill, J.R. (2002) Influence of Endurance Exercise Training Status and Gender on Postexercise Hypotension. Journal of Applied Physiology, 92, 2368-2374. https://doi.org/10.1152/japplphysiol.00020.2002

Submit or recommend next manuscript to SCIRP and we will provide best service for you:

Accepting pre-submission inquiries through Email, Facebook, LinkedIn, Twitter, etc. A wide selection of journals (inclusive of 9 subjects, more than 200 journals)

Providing 24-hour high-quality service

User-friendly online submission system

Fair and swift peer-review system

Efficient typesetting and proofreading procedure

Display of the result of downloads and visits, as well as the number of cited articles

Maximum dissemination of your research work

Submit your manuscript at: http://papersubmission.scirp.org/

Or contact health@scirp.org 